\title{
Adaptive Tracking Control of Uncertain MIMO Nonlinear Systems with Time-varying Delays and Unmodeled Dynamics
}

\author{
Xiao-Cheng Shi Tian-Ping Zhang \\ Department of Automation, College of Information Engineering, Yangzhou University, Yangzhou 225127, China
}

\begin{abstract}
In this paper, adaptive neural tracking control is proposed based on radial basis function neural networks (RBFNNs) for a class of multi-input multi-output (MIMO) nonlinear systems with completely unknown control directions, unknown dynamic disturbances, unmodeled dynamics, and uncertainties with time-varying delay. Using the Nussbaum function properties, the unknown control directions are dealt with. By constructing appropriate Lyapunov-Krasovskii functionals, the unknown upper bound functions of the time-varying delay uncertainties are compensated. The proposed control scheme does not need to calculate the integral of the delayed state functions. Using Young's inequality and RBFNNs, the assumption of unmodeled dynamics is relaxed. By theoretical analysis, the closed-loop control system is proved to be semi-globally uniformly ultimately bounded.
\end{abstract}

Keywords: Adaptive control, unmodeled dynamics, time-varying delays, neural networks, Nussbaum function

\section{Introduction}

During the past several decades, the design of adaptive controller for nonlinear systems has received much attention based on neural network (NN) approximation performance. Several stable adaptive NN control approaches were investigated $^{[1-3]}$. But there exist some types of matching conditions in most of these NN control schemes. Attempting to overcome these restrictions, adaptive backstepping design was presented for a class of strict-feedback nonlinear systems using neural network control ${ }^{[4]}$. Research on adaptive control techniques for multi-input multi-output (MIMO) nonlinear systems is of great significance. Using multirate sampled-data control technique, an adaptive output feedback control was proposed for a class of general MIMO systems ${ }^{[5]}$. Based on the principle of sliding mode control and using the properties of Nussbaum-type function, several adaptive neural control schemes were presented for a class of uncertain nonlinear state time delay systems $^{[6-8]}$. In addition, time delay uncertainties were compensated for by constructing appropriate LyapunovKrasovskii functionals in them. It is well known that time delays are often encountered in various systems, and the existence of time delays usually becomes the source of instability and makes the control synthesis more difficult. Lyapunov-Krasovskii functional in [9] and LyapunovRazumikhin functional in [10] are essential tools in the stability analysis of time-delay systems. Recently, several adaptive control schemes have been proposed that combined the Lyapunov-Krasovskii functional and the radial basis function neural networks (RBFNNs) or fuzzy logic systems for nonlinear systems with time delays ${ }^{[1-16]}$. In [11-13], three adaptive control schemes were developed for a class of nonlinear systems with constant delays. But time-varying delays often appeared in many systems ${ }^{[14-17]}$.

\footnotetext{
Manuscript received September 21, 2012; revised December 24, 2012 This work was supported by National Natural Science Foundation of China (No. 61174046).
}

Based on the principle of sliding mode control and using the integral-type Lyapunov function, adaptive control design was investigated for a class of uncertain MIMO state timevarying delay nonlinear systems with unknown disturbances and known gain signs ${ }^{[14]}$. In [15], by introducing Max function to construct continuous approximation function, a class of MIMO nonlinear systems with unknown dead-zone and time-varying delays was studied by variable structure control. Combining the separation technique with the dynamic surface control approach, and constructing an appropriate Lyapunov-Krasovskii functional, an adaptive dynamic surface control design was proposed for a class of nonlinear systems with unknown discrete and distributed time-varying delays ${ }^{[16]}$. Using the parameter separation technique and the Nussbaum-type function properties, a novel adaptive iterative learning control approach was proposed for a class of nonlinearly parameterized systems with unknown timevarying delay and unknown control direction ${ }^{[17]}$.

As we all known, unmodeled dynamics exists in many practical nonlinear systems, due to many factors, such as measurement noise, modeling errors, external disturbances, modeling simplifications, and they can severely degrade the closed-loop system performance. Therefore, various approaches were investigated to handle such systems with unmodeled dynamics ${ }^{[18-21]}$. In [18], a recursive robust adaptive control was proposed for a class of nonlinear systems with unmodeled dynamics by applying input-to-state stability property. However, the control gain and its direction are assumed to be known in the considered systems. Using Nussbaum-type functions and fuzzy logic systems, a direct adaptive robust control was presented for a class of singleinput single-output (SISO) nonlinear systems with unmodeled dynamics and unknown virtual control directions. The idea in $[18,19]$ was to use an available dynamic signal to conduct unmodeled dynamics. Combining backstepping with dynamic surface control, a novel adaptive control was presented for a class of nonlinear systems in pure feed- 
back form with unmodeled dynamics ${ }^{[20]}$. The assumptions with respect to unmodeled dynamics ${ }^{[20]}$ are similar to the work in [21] which satisfy a so-called lower-triangular condition. Tong et al. ${ }^{[18-21]}$ only considered unmodeled dynamics alone, but in practice, unmodeled dynamics and timevarying delays often appear simultaneously.

Motivated by the previous works, adaptive neural network control is investigated for a class of MIMO nonlinear systems in this paper. The main contributions of the paper are as follows.

1) Adaptive neural tracking control is proposed for a class of MIMO nonlinear systems with unmodeled dynamics, unknown dynamic disturbances, completely unknown control directions, and time-varying delay uncertainties. To the best of our knowledge, it is the first time to deal with such kind of systems with several uncertainties as compared to the existing literatures.

2) By constructing the appropriate Lyapunov-Krasovskii functionals in the control design, the assumptions with respect to the unmodeled dynamics and the dynamic disturbances as well as the time-varying delays are relaxed ${ }^{[6-8,12,14,15,20,21]}$. The upper bounds of the time-varying delay and the dynamic disturbance may be unknown. Moreover, the controller design does not calculate the time-varying gain using the functions of the upper bound of the time-varying delayed uncertainties $^{[6-8,12,14,15]}$.

This paper is organized as follows. The problem formulation and preliminaries are given in Section 2. In Section 3 , an adaptive RBFNN control is developed for a class of MIMO nonlinear systems by utilizing an integral-type Lyapunov function. Furthermore, the closed-loop system stability is analyzed as well. In Section 4, simulation results are demonstrated. Finally, Section 5 details the conclusions.

\section{Problem formulation and preliminar- ies}

\subsection{Problem formulation}

Consider a class of uncertain MIMO nonlinear systems with unmodeled dynamics and time-varying delay in the following form:

$$
\left\{\begin{aligned}
\dot{z}= & q(t, z, x) \\
\dot{x}_{1 j}= & x_{1, j+1}, j=1, \cdots, n_{1}-1 \\
\dot{x}_{1 n_{1}}= & f_{1}(x)+h_{1}\left(x_{\tau}\right)+b_{1}\left(x_{1}, \bar{x}_{2}^{+}, \cdots, \bar{x}_{m}^{+}\right) u_{1}+ \\
& \Delta_{1}(t, z, x) \\
\dot{x}_{i j}= & x_{i, j+1}, j=1, \cdots, n_{i}-1 \\
\dot{x}_{i n_{i}}= & f_{i}\left(x, u_{1}, \cdots, u_{i-1}\right)+b_{i}\left(\bar{x}_{i}, \bar{x}_{i+1}^{+}, \cdots, \bar{x}_{m}^{+}\right) u_{i}+ \\
& h_{i}\left(x_{\tau}\right)+\Delta_{i}(t, z, x), i=2,3, \cdots, m \\
y_{1}= & x_{11}, \cdots, y_{m}=x_{m 1}
\end{aligned}\right.
$$

where $z \in \mathbf{R}^{n_{0}}$ is the unmodeled dynamics, $x=$ $\left[x_{1}^{\mathrm{T}}, x_{2}^{\mathrm{T}}, \cdots, x_{m}^{\mathrm{T}}\right]^{\mathrm{T}} \in \mathbf{R}^{n}$ is the state vector, $x_{i}=$ $\left[x_{i 1}, \cdots, x_{i n_{i}}\right]^{\mathrm{T}}, \quad i=1, \cdots, m, n=\sum_{i=1}^{m} n_{i}, \quad u_{i} \in$ $\mathbf{R}, y_{i} \in \mathbf{R}$ are the $i$-th system's input and output, $\bar{x}_{i}=\left[x_{1}^{\mathrm{T}}, x_{2}^{\mathrm{T}}, \cdots, x_{i}^{\mathrm{T}}\right]^{\mathrm{T}}, \bar{x}_{i}^{+}=\left[x_{i 1}, \cdots, x_{i, n_{i}-1}\right]^{\mathrm{T}}, x_{\tau}=$ $\left[x_{1}^{\mathrm{T}}\left(t-\tau_{1}(t)\right), x_{2}^{\mathrm{T}}\left(t-\tau_{2}(t)\right), \cdots, x_{m}^{\mathrm{T}}\left(t-\tau_{m}(t)\right)\right]^{\mathrm{T}}, \tau_{i}(t), i=$ $1,2, \cdots, m$, are the unknown time-varying delays, $h_{i}\left(x_{\tau}\right)$, $f_{i}\left(x, u_{1}, \cdots, u_{i-1}\right)$ are the unknown continuous functions, $b_{i}\left(\bar{x}_{i}, \bar{x}_{i+1}^{+}, \cdots, \bar{x}_{m}^{+}\right)$are the unknown differentiable control gains, and $\Delta_{i}(t, z, x)$ denotes the unknown uncertain disturbance. For $t \in\left[-\tau_{\max }, 0\right], x(t)=\omega(t), \omega(t)$ is a known continuous initial state vector function. $\tau_{\max }$, which will be defined later, is an unknown positive constant.

The control objective is to design control $u_{i}$ for system (1) such that the output $y_{i}$ follows the desired trajectory $y_{i d}, i=1,2, \cdots, m$.

Define $x_{i d}, e_{i}$ and $s_{i}$ as

$$
\begin{aligned}
& x_{i d}=\left[y_{i d}, \dot{y}_{i d}, \cdots, y_{i d}^{\left(n_{i}-1\right)}\right]^{\mathrm{T}} \\
& e_{i}=x_{i}-x_{i d}=\left[e_{i 1}, e_{i 2}, \cdots, e_{i n_{i}}\right]^{\mathrm{T}} \\
& s_{i}=\left(\frac{\mathrm{d}}{\mathrm{d} t}+\lambda_{i}\right)^{n_{i}-1} e_{i 1}=\sum_{j=1}^{n_{i}-1} c_{i j} e_{i j}+e_{i n_{i}}
\end{aligned}
$$

where $c_{i j}=C_{n_{i}-1}^{j-1} \lambda_{i}^{n_{i}-j}, j=1,2, \cdots, n_{i}-1, \lambda_{i}>0, i=$ $1,2, \cdots, m$ are positive constants, specified by the designer.

Assumption 1. The desired trajectory vectors are continuous and available, and $\bar{x}_{i d}=\left[x_{i d}^{\mathrm{T}}, y_{i d}^{\left(n_{i}\right)}\right]^{\mathrm{T}} \in \Omega_{i d} \subset$ $\mathbf{R}^{n_{i}+1}, \Omega_{i d}$ is a known compact, $i=1,2, \cdots, m$.

Assumption 2. There exist unknown positive continuous functions $\rho_{i k}\left(x_{k}(t)\right)$ and unknown positive constants $\phi_{i}$ such as $\left|h_{i}\left(x_{\tau}\right)\right| \leqslant \phi_{i} \sum_{k=1}^{m} \rho_{i k}\left(x_{k}\left(t-\tau_{k}(t)\right)\right), \quad i=$ $1,2, \cdots, m$.

Assumption 3. The unknown state time-varying delays $\tau_{i}(t)$ satisfy $0 \leqslant \tau_{i}(t) \leqslant \tau_{\max }, \dot{\tau}_{i}(t) \leqslant \bar{\tau}_{\max }<1, i=$ $1,2, \cdots, m$, with the unknown constants $\tau_{\max }$ and $\bar{\tau}_{\max }$.

Assumption 4. Smooth functions $b_{i}\left(\bar{x}_{i}, \bar{x}_{i+1}^{+}, \cdots, \bar{x}_{m}^{+}\right)$ and their signs are unknown, and there exist constants $b_{i 0}$ and $b_{i 1}$ such that

$$
0<b_{i 0} \leqslant\left|b_{i}\left(\bar{x}_{i}, \bar{x}_{i+1}^{+}, \cdots, \bar{x}_{m}^{+}\right)\right| \leqslant b_{i 1}, \forall i=1,2 \cdots, m .
$$

Assumption 5. The equilibrium $z=0$ of system $\dot{z}=$ $q(t, z, 0)-q(t, 0,0)$ is globally asymptotically stable, i.e., there exists a Lyapunov function $W$ satisfying the following inequalities

$$
\begin{aligned}
& c_{1}\left\|\left.z\right|^{2} \leqslant W(t, z) \leqslant c_{2} \mid z\right\|^{2} \\
& \frac{\partial W}{\partial t}(t, z)+\frac{\partial W}{\partial z}(t, z)(q(t, z, 0)-q(t, 0,0)) \leqslant \\
& \quad-c_{3}\|z\|^{2}\left|\frac{\partial W}{\partial z}(t, z)\right| \leqslant c_{4}\|z\|
\end{aligned}
$$

where $c_{1}, c_{2}, c_{3}$, and $c_{4}$ are unknown positive constants, $\|\cdot\|$ denotes Euclidean norm. Also, there exists an unknown positive constant $c_{5}$ such that $\|q(t, 0,0)\| \leqslant c_{5}, \forall t \geqslant 0$.

Assumption 6. There exist unknown positive constant $p_{0}^{*}$ and unknown continuous function $\psi_{0} \in C^{1}, \psi_{0}(0)=0$ such that

$$
\|q(t, z, x)-q(t, z, 0)\| \leqslant p_{0}^{*} \psi_{0}(\|x\|) .
$$

Assumption 7. There exist unknown positive constants, $p_{i}^{*}$, such that $\forall(x, t) \in \mathbf{R}^{n_{i}} \times \mathbf{R}_{+}$

$$
\left|\Delta_{i}(t, z, x)\right| \leqslant p_{i}^{*} \rho_{i 1}(\|x\|)+p_{i}^{*}\|z\| \rho_{i 2}(x)
$$

where $\rho_{i 1}(\|x\|)$ and $\rho_{i 2}(x)$ are unknown positive continuous functions, $i=1, \cdots, m$. 
Remark 1. The functions $\rho_{i 1}(\|x\|)$ and $\rho_{i 2}(x)$ are unknown in this paper while they are known in [20,21]. In addition, $\psi_{0}(\|x\|)$ contains all the state variables. Compared with the existing literatures ${ }^{[20,21]}$, the assumptions with respect to dynamic disturbances and unmodeled dynamics are relaxed.

Lemma 1. (Young's inequality ${ }^{[22]}$ ) For vectors $x, y \in$ $\mathbf{R}^{n}$, the following inequality holds.

$$
x^{\mathrm{T}} y \leqslant \frac{\varepsilon^{p}\|x\|^{p}}{p}+\frac{\|y\|^{q}}{q \varepsilon^{q}}
$$

where $\varepsilon>0, p>1, q>1$, and $(p-1)(q-1)=1$.

\subsection{Radial basis function neural networks}

In this paper, we will employ radial basis function (RBF) neural networks to approximate unknown continuous function $h(Z): \mathbf{R}^{q} \rightarrow \mathbf{R}$ as discussed in $[23,24]$. Then, we have

$$
h(Z)=W^{* \mathrm{~T}} S(Z)+\delta(Z)
$$

where $S(Z)=\left[s_{1}(Z), s_{2}(Z), \cdots, s_{l}(Z)\right]^{\mathrm{T}} \in \mathbf{R}^{l}$ is the basis function vector, $s_{i}(Z)=\mathrm{e}^{-\frac{\left(Z-\mu_{i}\right)^{\mathrm{T}}\left(Z-\mu_{i}\right)}{k_{i}^{2}}}, i=$ $1,2, \cdots, l, \mu_{i}=\left[\mu_{i 1}, \mu_{i 2}, \cdots, \mu_{i q}\right]^{\mathrm{T}}$ is the center of the receptive field, $k_{i}$ is the width of the Gaussian function, and $W^{*}$ is an unknown ideal constant weight vector. It is defined as $W^{*}=\arg \min _{W \in \mathbf{R}^{l}} \sup _{Z \in \Omega_{Z}}\left\{\left|W^{\mathrm{T}} S(Z)-h(Z)\right|\right\}$, where $\delta(Z)$ is the approximation error, satisfying $|\delta(Z)| \leqslant \varepsilon$ with $\varepsilon$ being an unknown positive constant.

Choose $\lambda_{i}^{*}=\left\|W_{i}^{*}\right\|^{2}$ rather than $W_{i}^{*}$ as the parameter. $\hat{\lambda}_{i}$ is the estimation of the unknown constants $\lambda_{i}^{*}$, and the estimation error satisfies $\tilde{\lambda}_{i}=\lambda_{i}^{*}-\hat{\lambda}_{i}$.

\subsection{Nussbaum function properties}

In order to deal with the unknown control gain sign, the Nussbaum gain technique is employed in this paper. A function $N(\varsigma)$ is called Nussbaum-type function if it has the following properties:

$$
\begin{aligned}
& \text { 1) } \lim _{s \rightarrow+\infty} \sup \frac{1}{s} \int_{0}^{s} N(\varsigma) \mathrm{d} \varsigma=+\infty, \\
& \text { 2) } \lim _{s \rightarrow-\infty} \inf \frac{1}{s} \int_{0}^{s} N(\varsigma) \mathrm{d} \varsigma=-\infty .
\end{aligned}
$$

In this paper, $N(\varsigma)=\mathrm{e}^{\varsigma^{2}} \cos \left(\frac{\pi \varsigma}{2}\right)$ is used.

Lemma $2^{[8]}$. Let $V(\cdot)$ and $\varsigma(\cdot)$ be smooth functions defined on $\left[0, t_{f}\right)$ with $V(t) \geqslant 0, \forall t \in\left[0, t_{f}\right)$, and $N(\cdot)$ be an even smooth Nussbaum-type function. If the following inequality holds:

$$
\begin{gathered}
V(t) \leqslant \\
c_{0}+\mathrm{e}^{-c_{1} t} \int_{0}^{t} g(x(\tau)) N(\varsigma) \dot{\varsigma} \mathrm{e}^{c_{1} \tau} \mathrm{d} \tau+ \\
\mathrm{e}^{-c_{1} t} \int_{0}^{t} \dot{\varsigma} \mathrm{e}^{c_{1} \tau} \mathrm{d} \tau, \forall t \in\left[0, t_{f}\right)
\end{gathered}
$$

where $c_{0}$ represents some suitable constant, $c_{1}$ is a positive constant, and $g(x(\tau))$ is a time-varying parameter which takes values in the unknown closed intervals $I=\left[l^{-1}, l^{+1}\right]$, with $0 \notin I$, then $V(t), \varsigma(t)$ and $\int_{0}^{t} g(x(\tau)) N(\varsigma) \dot{\varsigma} \mathrm{d} \tau$ must be bounded on $\left[0, t_{f}\right)$.

\section{Control system design and stability analysis}

Consider the $i$-th subsystem, we obtain

$$
\begin{aligned}
\dot{s}_{i}= & \gamma_{i}+f_{i}\left(x, u_{1}, \cdots, u_{i-1}\right)+h_{i}\left(x_{\tau}\right)+ \\
& b_{i}\left(\bar{x}_{i}, \bar{x}_{i+1}^{+}, \cdots, \bar{x}_{m}^{+}\right) u_{i}+\Delta_{i}(t, z, x)
\end{aligned}
$$

where $\gamma_{i}=\sum_{j=1}^{n_{i}-1} c_{i j} e_{i, j+1}-y_{i d}^{\left(n_{i}\right)}$.

Define a smooth scalar function as

$$
V_{s_{i}}=\int_{0}^{s_{i}} \frac{\sigma}{\left|b_{i}\left(\bar{x}_{i-1}, \bar{x}_{i}^{+}, \sigma+\beta_{i}, \bar{x}_{i+1}^{+}, \cdots, \bar{x}_{m}^{+}\right)\right|} \mathrm{d} \sigma
$$

where $\beta_{i}=y_{i d}^{\left(n_{i}-1\right)}-\sum_{j=1}^{n_{i}-1} c_{i j} e_{i j}$. Obviously, we can conclude $\dot{\beta}_{i}=-\gamma_{i}$. By second mean value theorem for integrals, we can obtain $V_{s_{i}}=\frac{s_{i}^{2}}{2\left|b_{i}\left(\bar{x}_{i-1}, \bar{x}_{i}^{+}, \lambda_{s_{i}} s_{i}+\beta_{i}, \bar{x}_{i+1}^{+}, \cdots, \bar{x}_{m}^{+}\right)\right|}$ with $\lambda_{s_{i}} \in(0,1)$. Because $0<b_{i 0} \leqslant\left|b_{i}\left(\bar{x}_{i}, \bar{x}_{i+1}^{+}, \cdots, \bar{x}_{m}^{+}\right)\right|$, it is shown that $V_{s_{i}}$ is positive definite with respect to $s_{i}$.

Let $V_{w}=\frac{1}{\lambda_{0}} W$, where $\lambda_{0}$ is a positive constant, and $W$ is given in Assumption 1. Differentiating $V_{w}$ with respect to time $t$, we have

$$
\begin{aligned}
\dot{V}_{w}= & \frac{1}{\lambda_{0}}\left[\frac{\partial W}{\partial t}+\frac{\partial W}{\partial z} \dot{z}\right] \leqslant \\
& \frac{1}{\lambda_{0}}\left[-c_{3}\|z\|^{2}+c_{4} c_{5}\|z\|+c_{4} p_{0}^{*}\|z\| \psi_{0}(\|x\|)\right] \leqslant \\
& -\frac{1}{\lambda_{0}} c_{3}\|z\|^{2}+\frac{1}{8 \lambda_{0}} c_{3}\|z\|^{2}+\frac{2 \lambda_{0}}{c_{3}} c_{4}^{2} c_{5}^{2}+ \\
& \frac{1}{8 \lambda_{0}} c_{3}\|z\|^{2}+\frac{2 \lambda_{0} c_{4}^{2} p_{0}^{* 2} \psi_{0}(\|x\|)}{c_{3}} \leqslant \\
& -\frac{1}{\lambda_{0}} c_{3}\|z\|^{2}+\frac{1}{8 \lambda_{0}} c_{3}\|z\|^{2}+\frac{2 \lambda_{0}}{c_{3}} c_{4}^{2} c_{5}^{2}+ \\
& \frac{1}{8 \lambda_{0}} c_{3}\|z\|^{2}+\frac{\lambda_{0}^{2} c_{4}^{4} p_{0}^{* 4}}{c_{3}^{2}}+\psi_{0}^{4}(\|x\|) .
\end{aligned}
$$

For the sake of clarity and convenience, let

$$
\begin{aligned}
B_{i} & =\left|b_{i}\left(\bar{x}_{i}, \bar{x}_{i+1}^{+}, \cdots, \bar{x}_{m}^{+}\right)\right| \\
F_{i}\left(\sigma, \beta_{i}\right) & =\frac{1}{\left|b_{i}\left(\bar{x}_{i-1}, \bar{x}_{i}^{+}, \sigma+\beta_{i}, \bar{x}_{i+1}^{+}, \cdots, \bar{x}_{m}^{+}\right)\right|} \\
G_{j, k}^{i}\left(\sigma, \beta_{i}\right) & =\frac{\partial\left|b_{i}^{-1}\left(\bar{x}_{i-1}, \bar{x}_{i}^{+}, \sigma+\beta_{i}, \bar{x}_{i+1}^{+}, \cdots, \bar{x}_{m}^{+}\right)\right|}{\partial x_{j, k}} .
\end{aligned}
$$

Differentiating (6) with respect to time $t$, we obtain

$$
\begin{aligned}
\dot{V}_{s_{i}}= & \frac{s_{i}}{B_{i}} \dot{s}_{i}+\sum_{j=1}^{m} \sum_{k=1}^{n_{j}-1} \int_{0}^{s_{i}} \sigma G_{j, k}^{i}\left(\sigma, \beta_{i}\right) x_{j, k+1} \mathrm{~d} \sigma+ \\
& \sum_{j=1}^{i-1} \int_{0}^{s_{i}} \sigma G_{j, n_{j}}^{i}\left(\sigma, \beta_{i}\right) \dot{x}_{j, n_{j}} \mathrm{~d} \sigma+ \\
& \dot{\beta}_{i} \int_{0}^{s_{i}} \sigma \frac{\partial F_{i}\left(\sigma, \beta_{i}\right)}{\partial \beta_{i}} \mathrm{~d} \sigma .
\end{aligned}
$$


Substituting (5) into (7) and using $\dot{\beta}_{i}=-\gamma_{i}$, we obtain

$$
\begin{aligned}
\dot{V}_{s_{i}}= & \frac{s_{i}}{B_{i}}\left(f_{i}\left(x, u_{1}, \cdots, u_{i-1}\right)+\right. \\
& \left.h_{i}\left(x_{\tau}\right)+b_{i}\left(\bar{x}_{i}, \bar{x}_{i+1}^{+}, \cdots, \bar{x}_{m}^{+}\right) u_{i}+\Delta_{i}(t, z, x)\right)+ \\
& \sum_{j=1}^{m} \sum_{k=1}^{n_{j}-1} \int_{0}^{s_{i}} \sigma G_{j, k}^{i}\left(\sigma, \beta_{i}\right) x_{j, k+1} \mathrm{~d} \sigma+\int_{0}^{s_{i}} \gamma_{i} F_{i}\left(\sigma, \beta_{i}\right) \mathrm{d} \sigma+ \\
& \sum_{j=1}^{i-1} \int_{0}^{s_{i}} \sigma G_{j, n_{j}}^{i}\left(\sigma, \beta_{i}\right)\left[f_{j}\left(x, u_{1}, \cdots, u_{j-1}\right)+h_{j}\left(x_{\tau}\right)+\right. \\
& \left.b_{j}\left(\bar{x}_{j}, \bar{x}_{j+1}^{+}, \cdots, \bar{x}_{m}^{+}\right) u_{j}+\Delta_{j}(t, z, x)\right] \mathrm{d} \sigma .
\end{aligned}
$$

Applying Young's inequality, Assumptions 2 and 5, we obtain

$$
\begin{gathered}
\frac{s_{i}}{B_{i}} h_{i}\left(x_{\tau}\right) \leqslant \frac{1}{2} \sum_{k=1}^{m} \frac{\phi_{i}^{2} s_{i}^{2}}{B_{i}^{2}}+\frac{1}{2} \sum_{k=1}^{m} \rho_{i k}^{2}\left(x_{k}\left(t-\tau_{k}(t)\right)\right) \\
\sum_{j=1}^{i-1} \int_{0}^{s_{i}} \sigma G_{j, n_{j}}^{i}\left(\sigma, \beta_{i}\right) h_{j}\left(x_{\tau}\right) \mathrm{d} \sigma \leqslant \\
\frac{1}{2} \sum_{j=1}^{i-1} \sum_{k=1}^{m}\left(\int_{0}^{s_{i}} \sigma G_{j, n_{j}}^{i}\left(\sigma, \beta_{i}\right) \mathrm{d} \sigma\right)^{2} \phi_{j}^{2}+ \\
\frac{1}{2} \sum_{j=1}^{i-1} \sum_{k=1}^{m} \rho_{j k}^{2}\left(x_{k}\left(t-\tau_{k}(t)\right)\right) \\
\frac{s_{i} \Delta_{i}(t, z, x)}{B_{i}} \leqslant \frac{s_{i}^{2} \rho_{i 1}^{2}(\|x\|)}{2 a_{i i}^{2} B_{i}^{2}}+\frac{a_{i i}^{2} p_{i}^{* 2}}{2}+ \\
\frac{c_{3}\|z\|^{2}}{4 \lambda_{0}}+\frac{b_{i i}^{2} p_{i}^{* 4}}{2}+\frac{s_{i}^{4} \rho_{i 2}^{4}(x)}{2 b_{i i}^{2} B_{i}^{4}} \\
\sum_{j=1}^{i-1} \int_{0}^{s_{i}} G_{j, n_{j}}^{i}\left(\sigma, \beta_{i}\right) \sigma \Delta_{j}(t, z, x) \mathrm{d} \sigma \leqslant \\
\sum_{j=1}^{i-1}\left(\int_{0}^{s_{i}} \sigma G_{j, n_{j}}^{i}\left(\sigma, \beta_{i}\right) \mathrm{d} \sigma\right)^{2} \frac{\rho_{j 1}^{2}(\|x\|)}{2 a_{i j}^{2}}+ \\
\sum_{j=1}^{i-1} \frac{p_{j}^{* 2} a_{i j}^{2}}{2}+\frac{c_{3}\|z\|^{2}}{4 \lambda_{0}}+\sum_{j=1}^{i-1} \frac{b_{i j}^{2} p_{j}^{* 4}}{2}+ \\
\sum_{j=1}^{i-1}\left(\int_{0}^{s_{i}} \sigma G_{j, n_{j}}^{i}\left(\sigma, \beta_{i}\right) \mathrm{d} \sigma\right)^{4} \frac{\rho_{j 2}^{4}(x) \lambda_{0}^{2}}{2 b_{i j}^{2} c_{3}^{2}}
\end{gathered}
$$

where $a_{i j}, b_{i j}$ are positive constants.

Substituting (9)-(12) into (8) yields

$$
\begin{aligned}
\dot{V}_{s_{i}}+\dot{V}_{w} \leqslant & s_{i} g_{i}(t) u_{i}+s_{i} \varphi_{i}\left(Z_{i}\right)+ \\
& \frac{1}{2} \sum_{j=1}^{i} \sum_{k=1}^{m} \rho_{j k}^{2}\left(x_{k}\left(t-\tau_{k}(t)\right)\right)-\frac{c_{3}\|z\|^{2}}{4 \lambda_{0}}+ \\
& \psi_{0}^{4}(\|x\|)+\sum_{j=1}^{i} \frac{a_{i j}^{2} p_{j}^{* 2}}{2}+\sum_{j=1}^{i} \frac{b_{i j}^{2} p_{j}^{* 4}}{2}
\end{aligned}
$$

where

$$
\begin{aligned}
g_{i}(t) & =\frac{b_{i}\left(\bar{x}_{i}, \bar{x}_{i+1}^{+}, \cdots, \bar{x}_{m}^{+}\right)}{\left|b_{i}\left(\bar{x}_{i}, \bar{x}_{i+1}^{+}, \cdots, \bar{x}_{m}^{+}\right)\right|} \\
\varphi_{i}\left(Z_{i}\right)= & \frac{f_{i}\left(x, u_{1}, \cdots, u_{i-1}\right)}{B_{i}}+\sum_{k=1}^{m} \frac{\phi_{i}^{2} s_{i}}{2 B_{i}^{2}}+\frac{s_{i} \rho_{i 1}^{2}(\|x\|)}{2 a_{i i}^{2} B_{i}^{2}}+ \\
& \frac{s_{i}^{3} \rho_{i 2}^{4}(x)}{2 b_{i i}^{2} B_{i}^{4}}+s_{i} \sum_{j=1}^{m} \sum_{k=1}^{n_{j}-1} \int_{0}^{1} G_{j, k}^{i}\left(\sigma, \beta_{i}\right) \theta x_{j, k+1} \mathrm{~d} \theta+ \\
& \int_{0}^{1} \gamma_{i} F_{i}\left(\sigma, \beta_{i}\right) \mathrm{d} \theta+s_{i} \sum_{j=1}^{i-1} \int_{0}^{1} \theta G_{j, n_{j}}^{i}\left(\sigma, \beta_{i}\right) \times \\
& {\left[f_{j}\left(x, u_{1}, \cdots, u_{j-1}\right)+b_{j}\left(\bar{x}_{j}, \bar{x}_{j+1}^{+}, \cdots, \bar{x}_{m}^{+}\right) u_{j}\right] \mathrm{d} \theta+} \\
& \sum_{j=1}^{i-1} \sum_{k=1}^{m}\left[\int_{0}^{1} G_{j, n_{j}}^{i}\left(\sigma, \beta_{i}\right) \theta \mathrm{d} \theta\right]^{2} \frac{1}{2} \phi_{j}^{2} s_{i}^{3}+ \\
& \sum_{j=1}^{i-1} \frac{\rho_{j 1}^{2}(\|x\|) s_{i}^{3}}{2 a_{i j}^{2}}\left[\int_{0}^{1} \theta G_{j, n_{j}}^{i}\left(\sigma, \beta_{i}\right) \mathrm{d} \theta\right]^{2}+ \\
& \sum_{j=1}^{i-1}\left[\int_{0}^{1} \theta G_{j, n_{j}}^{i}\left(\sigma, \beta_{i}\right) \mathrm{d} \theta\right]^{4} \frac{\rho_{j 2}^{4}(x) \lambda_{0}^{2} s_{i}^{7}}{2 b_{i j}^{2} c_{3}^{2}} \\
& Z_{i}=\left[x^{\mathrm{T}}, s_{i}, \gamma_{i}, \beta_{i}, u_{1}, \cdots, u_{i-1}\right]^{\mathrm{T}} \in \Omega_{Z_{i} .}
\end{aligned}
$$

To overcome the design difficulties from the unknown time delay in (13), consider the following LyapunovKrasovskii functional

$$
V_{U_{i}}=\frac{\mathrm{e}^{-\gamma\left(t-\tau_{\max }\right)}}{2\left(1-\bar{\tau}_{\max }\right)} \sum_{j=1}^{i} \sum_{k=1}^{m} \int_{t-\tau_{k}(t)}^{t} \mathrm{e}^{\gamma \tau} \rho_{j k}^{2}\left(x_{k}(\tau)\right) \mathrm{d} \tau .
$$

Differentiating (15) with respect to $t$ and using (13) we have

$$
\begin{aligned}
\dot{V}_{s_{i}}+\dot{V}_{w}+\dot{V}_{U_{i}} \leqslant & \\
& s_{i}\left(g_{i}(t) u_{i}+h_{i}\left(Z_{i}\right)\right)-\frac{c_{3}\|z\|^{2}}{4 \lambda_{0}}+ \\
& \left(1-\frac{s_{i}^{2}}{c_{i}^{2}}\right) \psi_{0}^{4}(\|x\|)+\sum_{j=1}^{i} \frac{a_{i j}^{2} p_{j}^{* 2}}{2}+\sum_{j=1}^{i} \frac{b_{i j}^{2} p_{j}^{* 4}}{2}+ \\
& \frac{\mathrm{e}^{\gamma \tau_{\max }}\left(c_{i}^{2}-s_{i}^{2}\right)}{2\left(1-\bar{\tau}_{\max }\right) c_{i}^{2}} \sum_{j=1}^{i} \sum_{k=1}^{m} \rho_{j k}^{2}\left(x_{k}(t)\right)-\frac{\gamma}{2} V_{U_{i}}
\end{aligned}
$$

where

$$
\begin{aligned}
h_{i}\left(Z_{i}\right)= & \varphi_{i}\left(Z_{i}\right)+\frac{\mathrm{e}^{\gamma \tau_{\max }} s_{i}}{2\left(1-\bar{\tau}_{\max }\right) c_{i}^{2}} \sum_{j=1}^{i} \sum_{k=1}^{m} \rho_{j k}^{2}\left(x_{k}(t)\right)+ \\
& \frac{s_{i}}{c_{i}^{2}} \psi_{0}^{4}(\|x\|) .
\end{aligned}
$$

RBFNN $W_{i}^{* \mathrm{~T}} S_{i}\left(Z_{i}\right)$ in (3) is used to approximate the unknown continuous function $h_{i}\left(Z_{i}\right)$. From (3) and (16), and using the inequality $\delta_{i}\left(Z_{i}\right) s_{i} \leqslant \frac{1}{4} s_{i}^{2}+\varepsilon_{i}^{2}, W_{i}^{* \mathrm{~T}} S_{i}\left(Z_{i}\right) s_{i} \leqslant$ 
$\frac{\lambda_{i}^{*}}{2 \eta_{i}^{2}} S_{i}^{\mathrm{T}}\left(Z_{i}\right) S_{i}\left(Z_{i}\right) s_{i}^{2}+\frac{\eta_{i}^{2}}{2}$, we obtain

$$
\begin{aligned}
& \dot{V}_{s_{i}}+\dot{V}_{w}+\dot{V}_{U_{i}} \leqslant \\
& s_{i} g_{i}(t) u_{i}+\frac{\lambda_{i}^{*}}{2 \eta_{i}^{2}} S_{i}^{\mathrm{T}}\left(Z_{i}\right) S_{i}\left(Z_{i}\right) s_{i}^{2}+\frac{1}{4} s_{i}^{2}+ \\
& \varepsilon_{i}^{2}+\frac{\mathrm{e}^{\gamma \tau_{\max }}}{2\left(1-\bar{\tau}_{\max }\right)}\left(1-\frac{s_{i}^{2}}{c_{i}^{2}}\right) \sum_{j=1}^{i} \sum_{k=1}^{m} \rho_{j k}^{2}\left(x_{k}(t)\right)+ \\
& \quad \frac{\eta_{i}^{2}}{2}-\frac{c_{3}\|z\|^{2}}{4 \lambda_{0}}+\left(1-\frac{s_{i}^{2}}{c_{i}^{2}}\right) \psi_{0}^{4}(\|x\|)+ \\
& \sum_{j=1}^{i} \frac{a_{i j}^{2} p_{j}^{* 2}}{2}+\sum_{j=1}^{i} \frac{b_{i j}^{2} p_{j}^{* 4}}{2}-\frac{\gamma}{2} V_{U_{i}} .
\end{aligned}
$$

Consider the following control law

$$
\begin{aligned}
& u_{i}(t)=N\left(\varsigma_{i}\right)\left(k_{i} s_{i}+\frac{\hat{\lambda}_{i}}{2 \eta_{i}^{2}} S_{i}^{\mathrm{T}}\left(Z_{i}\right) S_{i}\left(Z_{i}\right) s_{i}\right) \\
& \dot{\zeta}_{i}=k_{i} s_{i}^{2}+\frac{\hat{\lambda}_{i}}{2 \eta_{i}^{2}} S_{i}^{\mathrm{T}}\left(Z_{i}\right) S_{i}\left(Z_{i}\right) s_{i}^{2}
\end{aligned}
$$

where $k_{i}>\frac{1}{4}$ is a positive constant, specified by the designer.

The adaptation law is given by

$$
\dot{\hat{\lambda}}_{i}=\frac{\omega_{i}}{2 \eta_{i}^{2}} S_{i}^{\mathrm{T}}\left(Z_{i}\right) S_{i}\left(Z_{i}\right) s_{i}^{2}-\sigma_{i} \hat{\lambda}_{i}
$$

where $\eta_{i}>0, \sigma_{i}>0$, and $\omega_{i}>0$.

Consider the following Lyapunov function candidate

$$
V_{i}=V_{s_{i}}+V_{w}+V_{U_{i}}+\frac{\tilde{\lambda}_{i}^{2}}{2 \omega_{i}} .
$$

Differentiating (21) with respect to $t$ yields

$$
\begin{aligned}
\dot{V}_{i} \leqslant & s_{i} g_{i}(t) u_{i}+\frac{\lambda_{i}^{*}}{2 \eta_{i}^{2}} S_{i}^{\mathrm{T}}\left(Z_{i}\right) S_{i}\left(Z_{i}\right) s_{i}^{2}+\frac{1}{4} s_{i}^{2}-\frac{\tilde{\lambda}_{i}}{\omega_{i}} \dot{\hat{\lambda}}_{i}+ \\
& \varepsilon_{i}^{2}+\frac{\mathrm{e}^{\gamma \tau_{\max }}}{2\left(1-\bar{\tau}_{\max }\right)}\left(1-\frac{s_{i}^{2}}{c_{i}^{2}}\right) \sum_{j=1}^{i} \sum_{k=1}^{m} \rho_{j k}^{2}\left(x_{k}(t)\right)+ \\
& \frac{\eta_{i}^{2}}{2}-\frac{c_{3}\|z\|^{2}}{4 \lambda_{0}}+\left(1-\frac{s_{i}^{2}}{c_{i}^{2}}\right) \psi_{0}^{4}(\|x\|)+ \\
& \sum_{j=1}^{i} \frac{p_{j}^{* 2}}{2}+\sum_{j=1}^{i} \frac{p_{j}^{* 4}}{2}-\frac{\gamma}{2} V_{U_{i}} .
\end{aligned}
$$

Substituting (18) and (19) into (22), and utilizing the inequality $\frac{\sigma_{i}}{\omega_{i}} \tilde{\lambda}_{i} \hat{\lambda}_{i} \leqslant-\frac{\sigma_{i}}{2 \omega_{i}} \tilde{\lambda}_{i}^{2}+\frac{\sigma_{i}}{2 \omega_{i}} \lambda_{i}^{* 2}$ yields

$$
\begin{aligned}
\dot{V}_{i} \leqslant & -\left(k_{i}-\frac{1}{4}\right) s_{i}^{2}-\frac{\sigma_{i}}{2 \omega_{i}} \tilde{\lambda}_{i}^{2}+g_{i}(t) N\left(\varsigma_{i}\right) \dot{\varsigma}_{i}+\dot{\varsigma}_{i}+ \\
& \frac{\sigma_{i}}{2 \omega_{i}} \lambda_{i}^{* 2}+\frac{\mathrm{e}^{\gamma \tau_{\max }}}{2\left(1-\bar{\tau}_{\max }\right)}\left(1-\frac{s_{i}^{2}}{c_{i}^{2}}\right) \sum_{j=1}^{i} \sum_{k=1}^{m} \rho_{j k}^{2}\left(x_{k}(t)\right)+ \\
& \frac{\eta_{i}^{2}}{2}+\varepsilon_{i}^{2}-\frac{c_{3}\|z\|^{2}}{4 \lambda_{0}}+\left(1-\frac{s_{i}^{2}}{c_{i}^{2}}\right) \psi_{0}^{4}(\|x\|)+ \\
& \sum_{j=1}^{i} \frac{a_{i j}^{2} p_{j}^{* 2}}{2}+\sum_{j=1}^{i} \frac{b_{i j}^{2} p_{j}^{* 4}}{2}-\frac{\gamma}{2} V_{U_{i}} .
\end{aligned}
$$

Remark 2. It is clearly seen from (23) that using appropriate Lyapunov-Krasovskii functionals not only effectively compensates for the delayed states, but also relaxes the assumption of the upper bound functions of time-varying delayed uncertainties. These upper bound functions are unknown continuous functions in this paper, while they must be known in $[6-8,12,14,15]$. Moreover, the controller $u_{i}$ does not require the delay integral term. This significantly alleviates the computational burden.

Define a compact set as

$$
\begin{aligned}
& \Omega_{z_{1}}=\left\{\left[x^{\mathrm{T}}, s_{1}, \gamma_{1}, \beta_{1}\right] \mid x_{j} \in \Omega_{j}, j=1, \cdots, m, \bar{x}_{1 d} \in \Omega_{1 d}\right\} \\
& \Omega_{z_{i}}=\left\{\left[x^{\mathrm{T}}, s_{i}, \gamma_{i}, \beta_{i}, u_{1}, \cdots, u_{i-1}\right]^{\mathrm{T}} \mid x_{j} \in \Omega_{j}, j=1, \cdots, m,\right. \\
& \left.\quad \bar{x}_{j d} \in \Omega_{j d}, j=1, \cdots, i\right\} \\
& \Omega_{i c_{i}}=\left\{x_{i}|| s_{i} \mid \leqslant c_{i}, \bar{x}_{i d} \in \Omega_{i d}\right\}, \quad i=1,2 \cdots, m
\end{aligned}
$$

where $\Omega_{j} \subset \mathbf{R}^{n_{j}}$ is a sufficiently large compact set, and $c_{i}$ is a positive constant that can be chosen arbitrarily small.

Theorem 1. Consider the closed-loop system consisting of the plant (1) under Assumptions 1-7, and the adaptive control given by (18)-(20). For bounded initial conditions, all the signals in the closed-loop system are semi-globally uniformly ultimately bounded, the parameter estimates

$$
\begin{aligned}
\hat{\lambda}_{i} \in & \left\{\hat{\lambda}_{i} \mid \tilde{\lambda}_{i}^{2} \leqslant 2 w_{i} \mu_{i}\right\} \\
x_{i} \in & \left\{x_{i} \mid\left\|x_{i}-x_{i d}\right\| \leqslant c_{i 0}\left(1+\left\|\Lambda_{i}\right\|\right)\left\|w_{i}(0)\right\|+\right. \\
& {\left.\left[1+\frac{\left(1+\left\|\Lambda_{i}\right\|\right) c_{i 0}}{\lambda_{i}}\right] \max \left\{\sqrt{2 b_{i 1} \mu_{i}}, c_{i}\right\}\right\} \subset \Omega_{i} . }
\end{aligned}
$$

Proof. Given the Lyapunov function candidate of (21), the proof includes the following three cases.

Case 1. $\left|s_{k}\right| \leqslant c_{k}, \forall k=1,2, \cdots, m$.

Because $\left|s_{k}\right| \leqslant c_{k}, \forall k=1,2, \cdots, m$ and $\rho_{j k}\left(x_{k}(t)\right)$ are continuous functions, we can let

$$
\begin{gathered}
\rho_{i, \max }=\sum_{j=1}^{i} \sum_{k=1}^{m} \max _{\left|s_{k}\right| \leqslant c_{k}} \rho_{j k}^{2}\left(x_{k}(t)\right) \\
\psi_{i, \max }=\max _{\substack{\left|s_{k}\right| \leqslant c_{k} \\
k=1, \cdots, m}} \psi_{0}^{4}(\|x\|) .
\end{gathered}
$$

From (23), we obtain

$$
\begin{aligned}
\dot{V}_{i} \leqslant & -\left(k_{i}-\frac{1}{4}\right) s_{i}^{2}-\frac{\gamma}{2} V_{U_{i}}-\frac{\sigma_{i}}{2 \omega_{i}} \tilde{\lambda}_{i}^{2}+g_{i}(t) N\left(\varsigma_{i}\right) \dot{\varsigma}_{i}- \\
& \frac{c_{3}\|z\|^{2}}{4 \lambda_{0}}+\dot{\varsigma}_{i}+\mu_{i 0}
\end{aligned}
$$

where

$$
\begin{aligned}
\mu_{i 0}= & \frac{\mathrm{e}^{\gamma \tau_{\max }}}{2\left(1-\bar{\tau}_{\max }\right)} \rho_{i, \max }+\frac{\eta_{i}^{2}}{2}+\varepsilon_{i}^{2}+\sum_{j=1}^{i} \frac{a_{i j}^{2} p_{j}^{* 2}}{2}+ \\
& \sum_{j=1}^{i} \frac{b_{i j}^{2} p_{j}^{* 4}}{2}+\frac{\sigma_{i}}{2 \omega_{i}} \lambda_{i}^{* 2}+\psi_{i}, \max .
\end{aligned}
$$

From (21) and (24), we obtain

$$
\dot{V}_{i}(t) \leqslant-\lambda_{i 0} V_{i}(t)+\mu_{i 0}+g_{i}(t) N\left(\varsigma_{i}\right) \dot{\varsigma}_{i}+\dot{\varsigma}_{i}
$$

where $\lambda_{i 0}=\min \left\{\left(2 k_{i}-0.5\right) b_{i 0}, \frac{\gamma}{2}, \sigma_{i}, \frac{c_{3}}{4 c_{2}}\right\}$.

From (25), we obtain

$$
V_{i}(t) \leqslant C_{i 0}+\mathrm{e}^{-\lambda_{i 0} t} \int_{0}^{t}\left(g_{i}(\tau) N\left(\varsigma_{i}\right)+1\right) \dot{\varsigma}_{i} \mathrm{e}^{\lambda_{i 0} \tau} \mathrm{d} \tau
$$


where $C_{i 0}=\frac{\mu_{i 0}}{\lambda_{i 0}}+V_{i}(0)$.

According to Lemma 1 , we have $V_{i}(t), \varsigma_{i}(t)$ and $\int_{0}^{t} g_{i}(\tau) N\left(\varsigma_{i}\right) \dot{\varsigma}_{i} \mathrm{~d} \tau$ are bounded on $\left[0, t_{f}\right]$. Therefore, $s_{i}$ and $\tilde{\lambda}_{i}$ are bounded on $\left[0, t_{f}\right]$. Let $C_{\varsigma_{i}}$ be the upper bound of $\mathrm{e}^{-\lambda_{i 0} t} \int_{0}^{t}\left(g_{i}(\tau) N\left(\varsigma_{i}\right)+1\right) \dot{\varsigma}_{i} \mathrm{~d} \tau$ on $[0,+\infty), \mu_{i}=C_{i 0}+C_{\varsigma_{i}}$, then $\tilde{\lambda}_{i}^{2} \leqslant 2 \mu_{i} \omega_{i}$ and $s_{i}^{2} \leqslant 2 b_{i 1} \mu_{i}$.

Define $w_{i}=\left[e_{i 1}, e_{i 2}, \cdots, e_{i n_{i}-1}\right]^{\mathrm{T}} \in \mathbf{R}^{n_{i}-1}$. From (2), we know that: 1) There is a state space representation for mapping $s_{i}=\left[\begin{array}{ll}\Lambda_{i}^{\mathrm{T}} & 1\end{array}\right] e_{i}$, i.e., $\dot{w}_{i}=A_{s_{i}} w_{i}+b_{s_{i}} s_{i}$ with $\Lambda_{i}=$ $\left[\lambda_{i 1}, \lambda_{i 2}, \cdots, \lambda_{i, n_{i}-1}\right]^{\mathrm{T}}, b_{s_{i}}=[0, \cdots, 0,1]^{\mathrm{T}} \in \mathbf{R}^{n_{i}-1}, A_{s_{i}}$ being a stable matrix. 2) There is a positive constant $c_{i 0}$ such that $\left\|\mathrm{e}^{A_{s_{i}} t}\right\| \leqslant c_{i 0} \mathrm{e}^{-\lambda_{i} t}$. 3) The solution for $w_{i}$ is

$$
\left.w_{i}(t)=\mathrm{e}^{A_{s_{i}}} w_{i}(0)+\int_{0}^{t} \mathrm{e}^{A_{s_{i}}(t-\tau}\right) b_{s_{i}} s_{i}(\tau) \mathrm{d} \tau .
$$

Accordingly, it follows that

$$
\left\|w_{i}(t)\right\| \leqslant c_{i 0}\left\|w_{i 0}\right\| \mathrm{e}^{-\lambda_{i} t}+c_{i 0} \int_{0}^{t} \mathrm{e}^{-\lambda_{i}(t-\tau)}\left|s_{i}(\tau)\right| \mathrm{d} \tau .
$$

Let $\bar{\mu}_{i}=\max \left\{\sqrt{2 b_{i 1} \mu_{i}}, c_{i}\right\}$. Therefore, we have

$$
\left\|w_{i}(t)\right\| \leqslant c_{i 0}\left\|w_{i}(0)\right\|+\frac{c_{i 0} \bar{\mu}_{i}}{\lambda_{i}} .
$$

Noting $s_{i}=\Lambda_{i}^{\mathrm{T}} w_{i}+e_{i n_{i}}, e_{i}=\left[w_{i}^{\mathrm{T}}, e_{i n_{i}}\right]^{\mathrm{T}}$, we obtain

$$
\left\|e_{i}\right\| \leqslant\left\|w_{i}\right\|+\left|e_{i n_{i}}\right| \leqslant\left(1+\left\|\Lambda_{i}\right\|\right) \| w_{i}+\left|s_{i}\right| .
$$

Substituting (27) into the above inequality leads to

$$
\left\|e_{i}\right\| \leqslant c_{i 0}\left(1+\left\|\Lambda_{i}\right\|\right)\left\|w_{i}(0)\right\|+\left[1+\frac{\left(1+\left\|\Lambda_{i}\right\|\right) c_{i 0}}{\lambda_{i}}\right] \bar{\mu}_{i} .
$$

Noting $x_{i}=e_{i}+x_{i d}$ and Assumption 1, we obtain

$$
\begin{gathered}
\left\|x_{i}\right\| \leqslant\left\|e_{i}\right\|+\left\|x_{i d}\right\| \leqslant c_{i 0}\left(1+\left\|\Lambda_{i}\right\|\right)\left\|w_{i}(0)\right\|+\left\|x_{i d}\right\|+ \\
{\left[1+\frac{\left(1+\left\|\Lambda_{i}\right\|\right) c_{i 0}}{\lambda_{i}}\right] \max \left\{\sqrt{2 b_{i 1} \mu_{i}}, c_{i}\right\} \in L_{\infty} .}
\end{gathered}
$$

Therefore, we can conclude from Case 1 that all the closedloop signals are semi-globally uniformly ultimately bounded for bounded initial conditions.

Case 2. $\left|s_{i}\right|>c_{i}$. From (24), we obtain

$$
\begin{gathered}
\dot{V}_{i} \leqslant-\left(k_{i}-\frac{1}{4}\right) s_{i}^{2}-\frac{\gamma}{2} V_{U_{i}}-\frac{\sigma_{i}}{2 \omega_{i}} \tilde{\lambda}_{i}^{2}+ \\
g_{i}(t) N\left(\varsigma_{i}\right) \dot{\varsigma}_{i}+\dot{\varsigma}_{i}+\mu_{i 0}
\end{gathered}
$$

where $\mu_{i 0}=\frac{\eta_{i}^{2}}{2}+\sum_{j=1}^{i} \frac{a_{i j}^{2} p_{j}^{* 2}}{2}+\sum_{j=1}^{i} \frac{b_{i j}^{2} p_{j}^{* 4}}{2}+\varepsilon_{i}^{2}+\frac{\sigma_{i}}{2 \omega_{i}} \lambda_{i}^{* 2}$. Similarly, we have $V_{i}(t) \leqslant \mu_{i}, \tilde{\lambda}_{i}^{2} \leqslant 2 \mu_{i} \omega_{i}$ and $s_{i}^{2} \leqslant 2 b_{i 1} \mu_{i}$.

Case 3. Let $\Sigma_{I}=\left\{i:\left|s_{i}\right| \leqslant c_{i}, i=1, \cdots, m\right\}$ and $\Sigma_{J}=\left\{j:\left|s_{j}\right|>c_{j}, j=1, \cdots, m\right\}$. From Case 2, for $j \in \Sigma_{J}$, we have $\left|s_{j}\right| \leqslant \sqrt{2 b_{j 1} \mu_{j}}$. Therefove, let

$$
\begin{aligned}
\rho_{i, \max }= & \sum_{j=1}^{i} \sum_{k \in \Sigma_{I}} \max _{\left|s_{k}\right| \leqslant c_{k}} \rho_{j k}^{2}\left(x_{k}(t)\right)+ \\
& \sum_{j=1}^{i} \sum_{k \in \Sigma_{J}\left|s_{k}\right| \leqslant \sqrt{2 b_{k 1} \mu_{k}}} \rho_{j k}^{2}\left(x_{k}(t)\right) \\
\psi_{i, \max }= & \max _{x \in \Omega} \psi_{0}(\|x\|)
\end{aligned}
$$

where

$$
\begin{gathered}
\Omega=\left\{x=\left[x_{1}^{\mathrm{T}}, \cdots, x_{m}^{\mathrm{T}}\right]^{\mathrm{T}}|| s_{i} \mid \leqslant c_{i}, i \in \Sigma_{I},\right. \\
\left.c_{j}<\left|s_{j}\right| \leqslant \sqrt{2 b_{j 1} \mu_{j}}, j \in \Sigma_{J}\right\} .
\end{gathered}
$$

From (23) and (29), we can also obtain (24). Thus, similarly, as discussed in Case 1, we can conclude that Theorem 1 holds.

\section{Simulation results}

To verify the effectiveness of the proposed approach, a numerical simulation is demonstrated.

Example 1. Consider the following MIMO nonlinear system with unmodeled dynamics and time-varying delays:

$$
\left\{\begin{aligned}
& \dot{z}=-z+0.25 x_{12}^{2} \sin (t) \\
& \dot{x}_{11}= x_{12} \\
& \dot{x}_{12}= x_{21}-0.3 \sin \left(x_{21}\right)+0.1 x_{11}^{2}\left(t-\tau_{1}(t)\right)+ \\
&\left(2-\sin ^{2}\left(x_{11}\right)\right) u_{1}+0.8\left(x_{11} x_{2}^{2}+\right. \\
&\left.x_{12}^{2}\right) \cos (1.5 t)+z^{2}+0.12 \sin (t) \\
& \dot{x}_{21}= x_{22} \\
& \dot{x}_{22}= x_{11}^{2} u_{1}+\left(x_{22}^{2}+x_{11}+0.5 \cos \left(x_{21}\right)\right) u_{1}^{2}+ \\
& 0.2 x_{22}\left(t-\tau_{2}(t)\right)+\sin \left(x_{21}\left(t-\tau_{2}(t)\right)\right)+ \\
&\left(3+\sin \left(x_{22}\right)\right) u_{2}+0.5\left(x_{11}^{2}+x_{21}^{2}\right) \sin (t)+ \\
& 2 z^{2}+0.2 \cos \left(0.5 x_{22}\right) \\
&= x_{11} \\
& y_{1} x_{21} \\
& y_{2}
\end{aligned}\right.
$$

where $u_{1}$ and $u_{2}$ are the control inputs. The control objective is to make the system outputs $y_{1}$ and $y_{2}$ follow the desired trajectories $y_{d 1}$ and $y_{d 2}$.

Neural networks $\hat{W}_{1}^{\mathrm{T}} S\left(Z_{1}\right)$ and $\hat{W}_{2}^{\mathrm{T}} S\left(Z_{2}\right)$ contain 50 and 60 nodes with centers taken randomly in the intervals $[-1.5,1.5]$, the widths are 2 and 4 . The design parameters of the above controller are $k_{1}=0.5, k_{2}=0.8, c_{1}=c_{2}=0.01$, $\eta_{1}=8, \eta_{2}=5, \sigma_{1}=0.0001, \sigma_{2}=0.001, \omega_{1}=8$, $\omega_{2}=10, \tau_{\max }=2$, and $\bar{\tau}_{\max }=0.5$. The initial condition is $\left[x_{11}(0), x_{12}(0), x_{21}(0), x_{22}(0), z(0)\right]^{\mathrm{T}}=[0,0,0,0,0]^{\mathrm{T}}$, time delays are $\tau_{1}(t)=0.2(1+\sin (t))$, and $\tau_{2}(t)=1-$ $0.5 \cos (t),\left[\hat{\lambda}_{1}(0), \hat{\lambda}_{2}(0)\right]^{\mathrm{T}}=[0,1]^{\mathrm{T}},\left[\varsigma_{1}(0), \varsigma_{2}(0)\right]^{\mathrm{T}}=[0,0]^{\mathrm{T}}$. Choose the desired tracking trajectories as

$y_{d 1}=0.5[\sin (t)+\sin (0.5 t)], y_{d 2}=\sin (0.5 t)+0.5 \sin (1.5 t)$.

The simulation results are shown in Figs. 1-9. The simulation results show that all the signals in the closed-loop control system are bounded and the approach of the proposed adaptive control is effective. Furthermore, from Figs.1-4, it can be seen that fairly good tracking performance is obtained. 


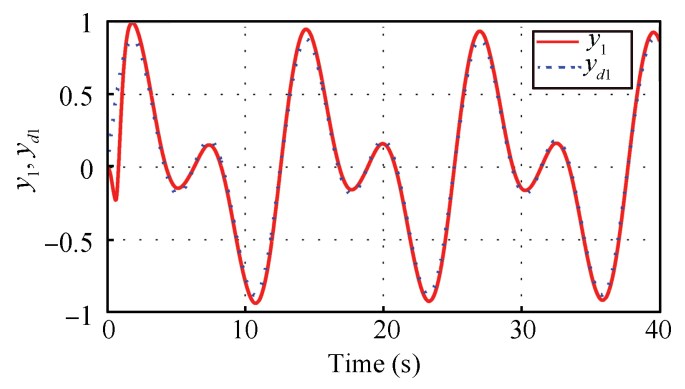

Fig. 1 Output $y_{1}$ and desired trajectory $y_{d 1}$

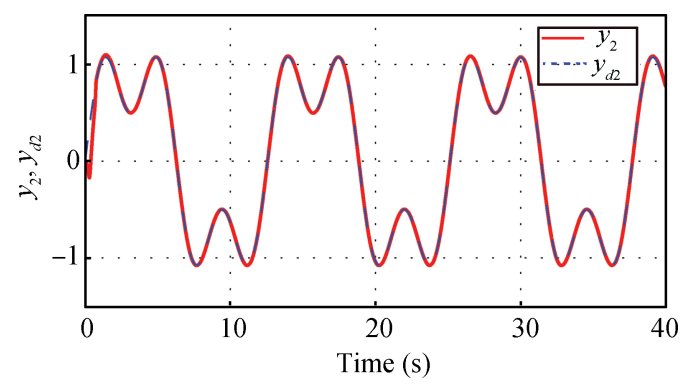

Fig. 2 Output $y_{2}$ and desired trajectory $y_{d 2}$

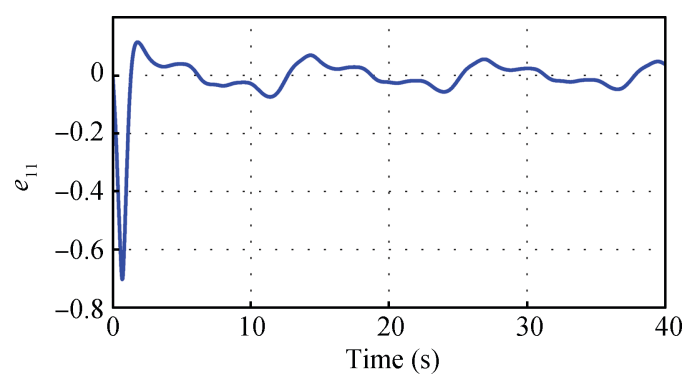

Fig. 3 Tracking error $e_{11}$

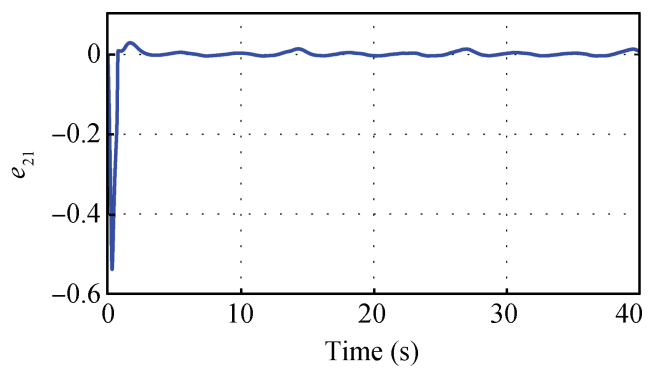

Fig. 4 Tracking error $e_{21}$

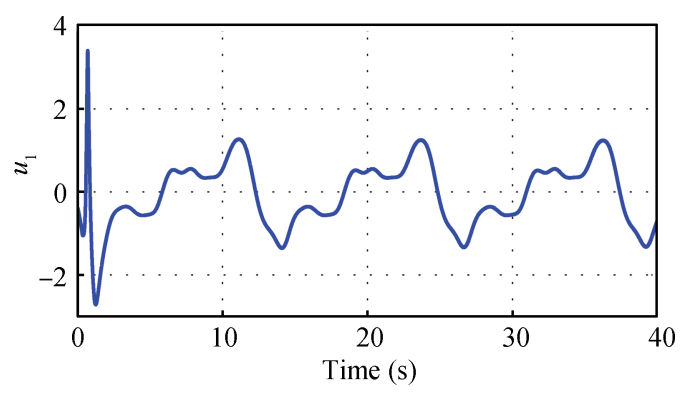

Fig. 5 Control signal $u_{1}$

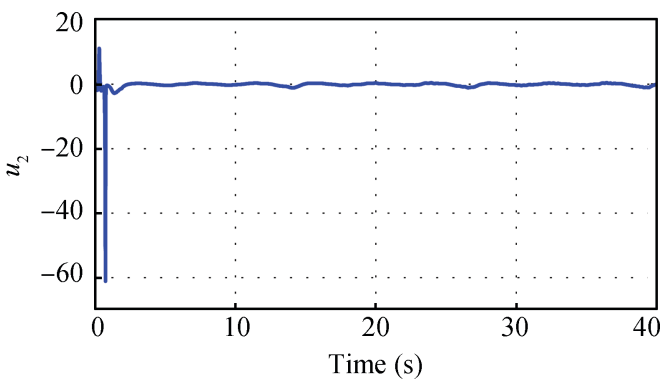

Fig. 6 Control signal $u_{2}$

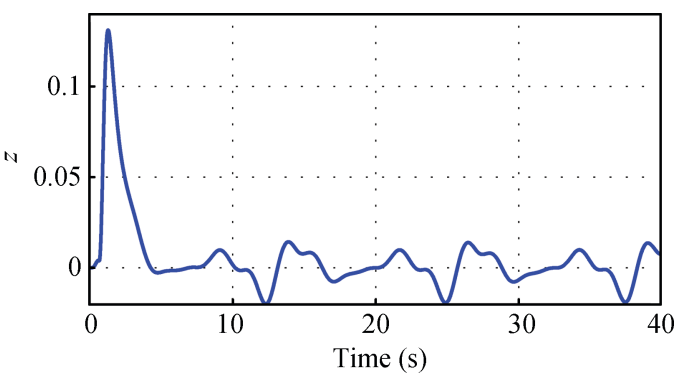

Fig. 7 Unmodeled dynamics $z$

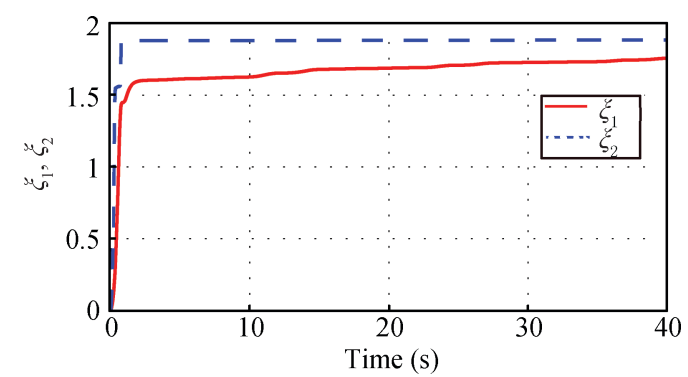

Fig. 8 Nussbaum tuning parameters $\zeta_{1}$ and $\zeta_{2}$

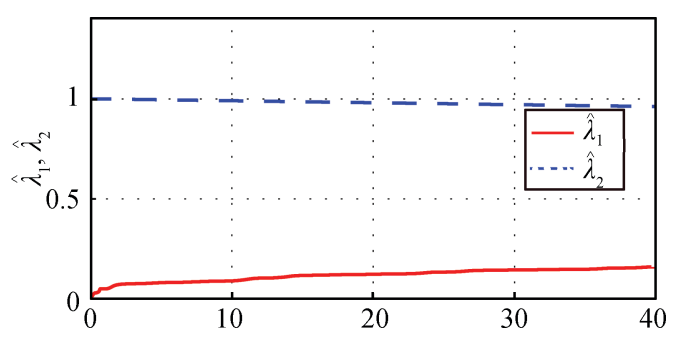

Fig. 9 Adaptive adjusting parameters $\hat{\lambda}_{1}$ and $\hat{\lambda}_{2}$

\section{Conclusions}

Adaptive neural tracking control has been proposed for a class of MIMO time-varying delay nonlinear systems with completely unknown virtual control directions, unknown uncertain dynamic disturbances, and unmodeled dynamics. RBFNNs have been employed to approximate unknown continuous functions. The restrictions of unmodeled dynamics are relaxed by utilizing Young's inequality. Based on Lyapunov theory, the closed-loop system is proved to be semi-globally uniformly ultimately bounded. In the future work, we will further relax the restriction of the timevarying delays uncertainties and extend the adaptive neural 
tracking control to a class of MIMO stochastic nonlinear systems.

\section{References}

[1] S. S. Ge, C. C. Huang, T. Zhang. Adaptive neural network control of nonlinear systems by state and output feedback. IEEE Transactions on Systems, Man, and Cybernetics Part B: Cybernetics, vol. 29, no. 6, pp. 818-828, 1999.

[2] S. S. Ge, C. Wang, T. H. Lee. Adaptive backstepping control of a class of chaotic systems. International Journal of Bifurcation and Chaos, vol. 10, no. 5, pp. 1149-1156, 2000.

[3] S. S. Ge, C. C. Huang, T. Zhang. Nonlinear adaptive control using neural networks and its application to CSTR systems. Journal of Process Control, vol. 9, no. 4, pp. 313-323, 1999.

[4] T. Zhang, S. S. Ge, C. C. Hang. Adaptive neural network control for strict-feedback nonlinear systems using backstepping design. Automatica, vol. 36, no. 12, pp. 1835-1846, 2000.

[5] I. Mizumoto, T. W Chen, S. Ohdaira, K. Makoto, I. Zenta. Adaptive output feedback control of general MIMO systems using multirate sampling and its application to a cart-crane system. Automatica, vol. 43, no. 12, pp. 2077-2085, 2007.

[6] T. P. Zhang, S. S. Ge. Adaptive neural control of MIMO nonlinear state time-varying delay systems with unknown dead-zones and gain signs. Automatica, vol.43, no. 6 , pp. 1021-1033, 2007.

[7] H. B. Qian, T. P. Zhang. Adaptive control of MIMO nonlinear time-varying delay systems with unknown gain signs. Control and Decision, vol. 23, no. 10, pp. 1153-1158, 2008. (in Chinese)

[8] S. S. Ge, F. Hong, T. H. Lee. Adaptive neural control of nonlinear time-delay systems with unknown virtual control coefficients. IEEE Transactions on Systems, Man, and Cybernetics - Part B: Cybernetics, vol.34, no. 1, pp. 499-516, 2004.

[9] J. Hale. Theory of Functional Differential Equations, New York: Springer, 1977.

[10] M. Jankovic. Control Lyapunov-Razumikhin functions and robust stabilization of time delay systems. IEEE Transactions on Automatic Control, vol.46, no. 7, pp. 1048-1060, 2001.

[11] S. S. Ge, F. Hong, T. H. Lee. Robust adaptive control of nonlinear systems with unknown time delays. Automatica, vol. 41, no. 7, pp. 1181-1190, 2005.

[12] S. S. Ge, K. P. Tee. Approximation-based control of nonlinear MIMO time-delay systems. Automatica, vol.43, no. 1, pp. 31-43, 2007.

[13] M. Wang, B. Chen, K. F. Liu, S. Y. Zhang. Adaptive fuzzy tracking control of nonlinear time-delay systems with unknown virtual control coefficients. Information Sciences, vol. 178, no. 22, pp. 4326-4340, 2008.

[14] Q. Q. Zhu, T. P. Zhang, B. C. Zhu. Adaptive control of MIMO nonlinear systems with disturbances and time delays. In Proceedings of the Chinese Control and Decision Conference, IEEE, Xuzhou, China, pp. 199-204, 2010.

[15] T. P. Zhang, C. Y. Zhou, Q. Zhu. Adaptive variable structure control of MIMO nonlinear systems with time-varying delays and unknown dead-zones. International Journal of Automation and Computing, vol.6, no. 2, pp.124-136, 2009 .
[16] H. Y. Yue, J. M. Li. Adaptive fuzzy dynamic surface control for a class of perturbed nonlinear time-varying delay systems with unknown dead-zone. International Journal of Automation and Computing, vol. 9, no. 5, pp. 545-554, 2012.

[17] D. Li, J. M. Li. Adaptive iterative learning control for nonlinearly parameterized systems with unknown time-varying delay and unknown control direction. International Journal of Automation and Computing, vol.9, no.6, pp.578-586, 2012 .

[18] S. C. Tong, X. L. He, Y. M. Li, H. G. Zhang. Adaptive fuzzy backstepping robust control for uncertain nonlinear systems based on small-gain approach. Fuzzy Sets and Systems, vol. 161, no. 6, pp. 771-796, 2010.

[19] S. C. Tong, Y. M. Li. Fuzzy adaptive robust backstepping stabilization for SISO nonlinear systems with unknown virtual control direction. Information Sciences, vol. 180, no. 23, pp. 4619-4640, 2010.

[20] T. P. Zhang, Y. Lu. Adaptive dynamic surface control of nonlinear systems with unmodeled dynamics. Control and Decision, vol. 27, no. 3, pp. 335-342, 2012. (in Chinese)

[21] Z. P. Jiang, D. J. Hill. A robust adaptive backstepping scheme for nonlinear systems with unmodeled dynamics. IEEE Transactions on Automatic Control, vol.44, no.9, pp. 1705-1711, 1999.

[22] M. Krstic, I. Kanellakopoulos, P. V. Kokotovic. Nonlinear and Adaptive Control Design, New York: Wiley, 1995.

[23] R. M. Sanner, J. J. E. Slotine. Gaussian networks for direct adaptive control. IEEE Transactions on Neural Networks, vol. 3, no. 6, pp. 837-863, 1992.

[24] S. S. Ge, C. C. Hang, T. H. Lee, T. Zhang. Stable Adaptive Neural Network Control, Boston: Kluwer Academic, pp. $27-46,2001$.

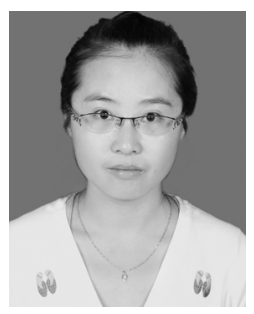

Xiao-Cheng Shi received the B. Eng. degree in automation from Yangzhou University, China in 2010. She is currently a master student in control theory and control engineering at Yangzhou University, China.

Her research interests include robust adaptive control, dynamic surface control, and nonlinear control.

E-mail: xiaocheng_shi@163.com

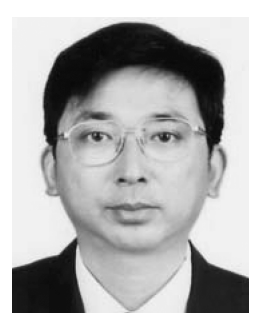

Tian-Ping Zhang received the B.Sc. degree in mathematics from Yangzhou Teachers College, Yangzhou, China in 1986, the M.Sc. degree in operations research and control theory from East China Normal University, Shanghai, China in 1992, and the $\mathrm{Ph}$. D. degree in automatic control theory and applications from Southeast University, Nanjing, China in 1996. He is now a professor in the College of Information Engineering, Yangzhou University, Yangzhou, China. From 2005 to 2006, he was a visiting scientist in the Department of Electrical and Computer Engineering, National University of Singapore, Singapore. He has published more than 180 papers in journals and conferences.

His research interests include fuzzy control, adaptive control, intelligent control, and nonlinear control.

E-mail: tpzhang@yzu.edu.cn (Corresponding author) 